\title{
Prevalence of CYP2B6 polymorphisms in Argentinians: the role of genetic testing
}

\author{
P. Scibona ${ }^{1,2}$, C. Vazquez ${ }^{1}$, A.R. Cajal' ${ }^{2}$, P.F. Argibay ${ }^{2}$ and W.H. Belloso ${ }^{1}$ \\ ${ }^{1}$ Clinical Pharmacology Section, Internal Medicine Service, \\ Hospital Italiano de Buenos Aires, Buenos Aires, Argentina \\ ${ }^{2}$ Instituto de Ciencias Básicas y Medicina Experimental, \\ Hospital Italiano de Buenos Aires, Buenos Aires, Argentina \\ Corresponding author: P. Scibona \\ E-mail: paula.scibona@hospitalitaliano.org.ar \\ Genet. Mol. Res. 14 (4): 16594-16599 (2015) \\ Received August 11, 2015 \\ Accepted October 4, 2015 \\ Published December 11, 2015 \\ DOI http://dx.doi.org/10.4238/2015.December.11.6
}

ABSTRACT. CYP2B6 is a highly polymorphic isoenzyme involved in the metabolism of many drugs including cyclophosphamide, bupropion, and efavirenz. A single nucleotide polymorphism (SNP) in CYP2B6 (516G>T) resulted in decreased expression and function associated with the CYP2B6*6 haplotype. Among the clinical implications of this phenotype, decreased activation of cyclophosphamide and increased plasma levels of efavirenz associated with increased central nervous system toxicity have been reported. The frequency of the CYP2B6 (516G>T) SNP has been studied in several different populations, but there is no data regarding distribution among Argentinians. In this study, 102 DNA samples from healthy volunteers were analyzed using a polymerase chain reactionrestriction fragment length polymorphism reaction specific for the CYP2B6 $(516 G>T)$ SNP. Our results showed a prevalence of $71.08 \%$ for the $G$ allele and $28.92 \%$ for the $T$ allele. This was distributed as $52.9 \%$ for the GG genotype (reduced dosage required), $36.6 \%$ for the GT genotype (normal dosage range), and $10.8 \%$ for the TT genotype (high drug toxicity). There was no preferential gender distribution observed. The relatively high 
prevalence of the TT genotype in our population supports the clinical use of genotyping as an additional tool in personalized medicine.

Key words: CYP2B6; CYP2B6*6 haplotype; 516G>T; Efavirenz; Bupropion; Cyclophosphamide

\section{INTRODUCTION}

More than $90 \%$ of phase I drug metabolism relies on cytochrome P450 (CYP) enzymes. Among the 59 active enzymes in the human CYP superfamily, CYP2B6 was initially thought to only have a minor role, accounting for a very small fraction of drug biotransformation (Wang and Tompkins, 2008). Nevertheless, CYP2B6 is of particular interest as it is involved in the metabolism of clinically relevant drugs such as cyclophosphamide, a commonly prescribed immunosuppressant that depends on CYP2B6 for its pharmacological activation to 4-hydroxycyclophosphamide (Raccor et al., 2012); bupropion, an antidepressant widely used in smoking cessation therapeutic strategies; and efavirenz (and to a lesser extent also nevirapine), a non-nucleoside reverse HIV transcriptase inhibitor that is part of the initial treatment regimen of patients with HIV and is used in conjunction with nucleoside/nucleotide reverse transcriptase inhibitors (Panel on Antiretroviral Guidelines for Adults and Adolescents 2012, 2013). The CYP2B6 gene is highly polymorphic. Among CYP2B6 gene variants, the CYP2B6* 6 haplotype is associated with lower mRNA levels, lower protein expression, and decreased bupropion hydroxylase activity compared with wild-type CYP2B6*1, particularly in the liver. The CYP2B6*6 haplotype is characterized by the presence of two non-synonymous single nucleotide polymorphisms (SNPs): $516 \mathrm{G}>\mathrm{T}$ and $785 \mathrm{~A}>\mathrm{G}$. These variants occur with or without additional promoter variants $\left(C Y P 2 B 6^{*} 6 A: 516 \mathrm{G}>\mathrm{T}\right.$ and $785 \mathrm{~A}>\mathrm{G}$; CYP2B6*6B: $516 \mathrm{G}>\mathrm{T}, 785 \mathrm{~A}>\mathrm{G},-1456 \mathrm{~T}>\mathrm{C}$ and $-750 \mathrm{~T}>\mathrm{C}$, and $\mathrm{CYP} 2 \mathrm{~B} 66^{*} 6 \mathrm{C}: 516 \mathrm{G}>\mathrm{T}, 785 \mathrm{~A}>\mathrm{G}$ and $-750 \mathrm{~T}>\mathrm{C}$ ). The functional consequences of $C Y P 2 B 6^{*} 6$ have been recently linked to an aberrant splicing variant determined by $516 \mathrm{G}>\mathrm{T}$ SNP at the region including exons 4 to 6 , leading to decreased expression and function associated with the CYP2B6*6 haplotype (Hofmann et al., 2008).

Efavirenz is a drug with a narrow therapeutic range and its acceptable plasma concentration at 12 hours is $1-4 \mu \mathrm{g} / \mathrm{mL}$ (Marzolini et al., 2001; Panel on Antiretroviral Guidelines for Adults and Adolescents 2012, 2013). Several studies have shown that SNPs in CYP2B6 -particularly $516 \mathrm{G}>\mathrm{T}$ - are associated with high plasma concentrations of efavirenz and drug-related toxicity (Nyakutira et al., 2008, Ramachandran et al., 2009, Aurpibul et al., 2012). In addition, the CYP2B6 polymorphism 516G $>\mathrm{T}$ was shown to be associated with higher than expected concentrations of the intravenous anesthetic propofol (Mastrogianni et al., 2014). The GT genotype results in normal drug metabolism while the GG genotype requires reduced doses of these drugs and the TT genotype results in higher risk of drug toxicity.

The frequencies of different CYP2B6 polymorphisms have been studied in different patient populations, showing a highly variable distribution (Arnaldo et al., 2013). Specifically for the $516 \mathrm{G}>\mathrm{T}$ polymorphism, the global distribution for the $\mathrm{G}$ and $\mathrm{T}$ alleles is 73 and $26 \%$, respectively (genotypes GG: $54.1 \%$, GT: $38.4 \%$, TT: $7.5 \%$ ). In Europe, the distribution is G: $76.8 \%$, T: $23.2 \%$ (genotypes GG: $57.8 \%$, GT: $38 \%$, TT: $4.2 \%$ ), in the United States of America it is G: $63.8 \%$, T: 36.2\% (genotypes GG: $40.9 \%$, GT: $45.9 \%$, TT: $13.3 \%$ ), and in Africa, which has the highest prevalence of the polymorphism, it is G: $65 \%$, T: $35 \%$ (genotypes GG: $42.3 \%$, GT: $44.7 \%$, TT: $13 \%$ ) (1000 Genomes Project Consortium et al., 2012). 
The majority of the inhabitants of Argentina are of Caucasian descent so the distribution of the CYP2B6 genotypes would be expected to be similar to that of Europe. However, there is no formal data about $C Y P 2 B 6$ allelic frequencies in Argentina. The aim of the present study is to determine the frequency of the most common CYP2B6 SNP, 516G>T, in an Argentinian population.

\section{MATERIAL AND METHODS}

\section{Study population}

One hundred and two random samples from healthy controls obtained from the Hospital Italiano de Buenos Aires DNA Bank were analyzed. Informed consent was obtained from all participants and approval was received from the appropriate institutional review boards.

\section{Sample collection and DNA extraction}

Peripheral blood $(10 \mathrm{~mL})$ was collected from all patients in 5-mL EDTA tubes. Whole-blood samples were stored at $4^{\circ} \mathrm{C}$ until the time of processing. Genomic DNA was extracted and purified using the QIAmp DNA Blood Mini kit (Qiagen, USA).

\section{Genotyping of CYP2B6}

Genotyping of the CYP2B6 516 allele was performed by polymerase chain reactionrestriction fragment length polymorphism (PCR-RFLP). Genomic DNA was amplified using sequence-specific primers for exon 4 of the CYP2B6 gene, generating a 509-bp amplicon. The PCR was performed in a $20-\mu \mathrm{L}$ reaction volume containing 1 X PCR buffer (Promega, Biodynamics Distributor, CABA, Buenos Aires, Argentina), 200 mM dNTPs (Fermentas, CABA, Buenos Aires, Argentina), 0.2 M each primer (Eurofins MWG Operon, Huntsville, AL, USA), 1 Unit Taq polymerase (Promega) and $100 \mathrm{ng}$ genomic DNA. Reactions were performed in a MJ Research Thermocycler (PTC 100). Cycling parameters were as follows: initial denaturation at $95^{\circ} \mathrm{C}$ for 5 min, and 30 cycles of $96^{\circ} \mathrm{C}$ for $30 \mathrm{~s}, 55^{\circ} \mathrm{C}$ for $30 \mathrm{~s}$, and $72^{\circ} \mathrm{C}$ for $40 \mathrm{~s}$, and a final extension step at $72^{\circ} \mathrm{C}$ for $10 \mathrm{~min}$. Aliquots of the PCR product were digested with restriction enzymes Bsrl (Avall) (Fermentas), at $65^{\circ} \mathrm{C}$ overnight to ensure complete digestion. The DNA fragments were electrophoresed on a $3 \%$ agarose gel stained with ethidium bromide, and bands were visualized with a UV transilluminator.

\section{Statistical analysis}

Allelic and genotypic frequencies were expressed in absolute and percentage values in the study population. Sample size calculation for estimating the TT genotype in the European population with a prevalence of $4 \%$ gives a sample size of 64 samples and a prevalence of $7 \%$ of the global population gives a sample size of 110 samples. The level of significance ( $\alpha$ error) was established at $5 \%$. The results obtained were further evaluated for the Hardy-Weinberg equilibrium in order to validate the finding. 


\section{RESULTS}

All patient samples were genotyped for the CYP2B6 516 allele according to methods previously described. PCR-RFLP analysis with digestion of the $509 \mathrm{bp}$ amplicon of CYP2B6 with $B s r l$ resulted in two fragments of 268 and 241 bp for the GG genotype and a single undigested 509 bp fragment for the TT genotype. The 3 fragments were present for the GT genotype (Figure 1). Among 102 subjects analyzed, $45.1 \%$ (46) were males and $54.9 \%(56)$ were females with a median age of 32 and an age range of 21 to 80 . In our study population, distribution of the CYP2B6 $516 \mathrm{G}>\mathrm{T}$ allele was as follows: $71.08 \%$ for the $\mathrm{G}$ allele and $28.92 \%$ for the $\mathrm{T}$ allele, and $52.9 \%$ (54) had the GG genotype, 36.6\% (37) had the GT genotype, and 10.8\% (11) had the TT genotype (Figure 2). There was no preferential gender distribution observed between genotypes.

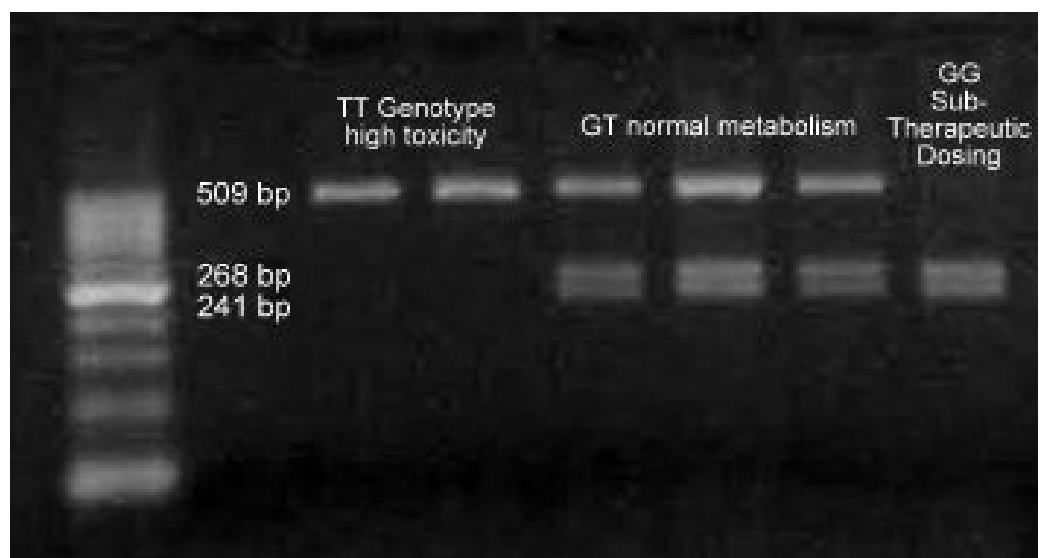

Figure 1. CYP2B6 516 genotyping using restriction enzyme Bsrl and visualization of results by gel electrophoresis.

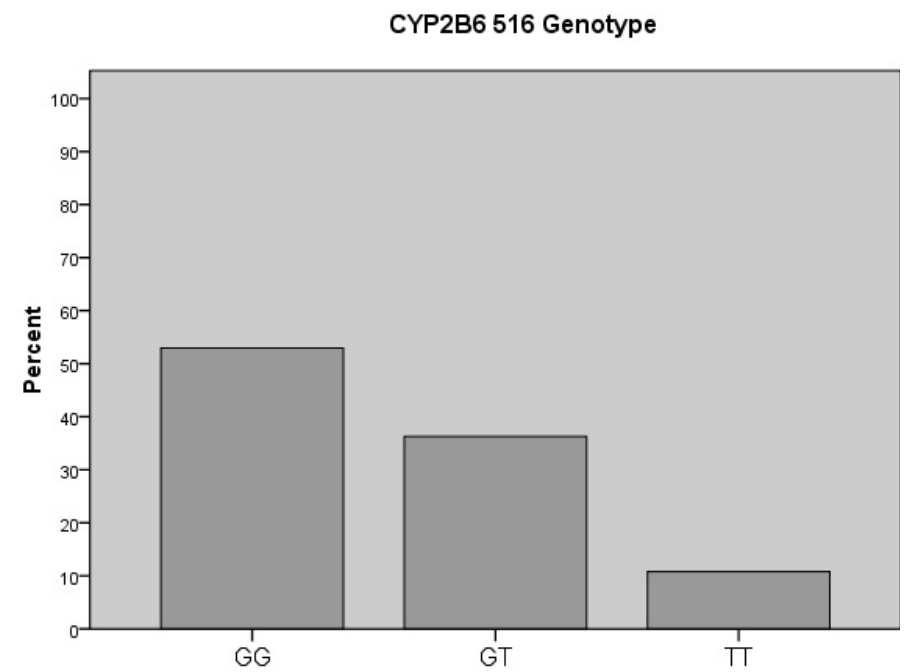

Figure 2. CYP2B6 516 genotype distributions. 


\section{DISCUSSION}

A significant proportion of inter-individual variability in the response to CYP2B6 substrates (such as efavirenz) has been attributed to the presence of a SNP at position 516 (G>T), although the effect varies between populations in relation to differential frequencies of these genetic variants. Thus, it has become important to perform frequency studies of potentially relevant polymorphisms in different populations in order to establish the relative importance of specific allelic variants and to estimate the potential usefulness of genetic analysis in a clinical scenario.

In the present study, we analyzed the frequency of the allelic variants for CYP2B6 516 in the Argentinian population. The prevalence of TT genotype was $10.8 \%$, which is double than its frequency in European populations (4.2\%) and similar to the frequency found in Native Americans and persons of African descent (13.3 and 13\%, respectively).

Contemporary Argentinians carry components of European, Native American and African ancestry but the relative amount depends on the genetic subsystem examined. Argentinians carry a large fraction of European genetic heritage in their Y-chromosome (94.1\%) and autosomal $(78.5 \%)$ DNA, but the mitochondrial gene pool is mostly of Native American ancestry (53.7\%). African heritage is relatively small in all three genetic subsets (<4\%) (Corach et al., 2010).

Since most Argentinian inhabitants are of Caucasian descent, a European genotype frequency profile is to be expected. However, the contribution of Native American ancestry to particular gene pool components may explain in part the higher prevalence of the TT genotype in CYP2B6 in our population in comparison with the expected $4.2 \%$ observed in European studies. This finding provides additional information to the ongoing controversy regarding which population-if any-can be used as a general reference for Argentinian genotypic profiles. In addition, the relatively high prevalence of homozygous variants supports the use of this analysis in clinical practice as an additional tool for dose customization of substrate medications.

\section{Conflicts of interest}

The authors declare no conflict of interest.

\section{REFERENCES}

Arnaldo P, Thompson RE, Lopes MQ, Suffys PN, et al. (2013). Frequencies of Cytochrome P450 2B6 and 2C8 Allelic Variants in the Mozambican Population. Malays. J. Med. Sci. 20: 13-23.

Aurpibul L, Chotirosniramit N, Sugandhavesa P, Kosashunhanan N, et al. (2012). Correlation of CYP2B6-516G > T Polymorphism with Plasma Efavirenz Concentration and Depression in HIV-Infected Adults in Northern Thailand. Curr. HIV Res. 10: 653-660.

Corach D, Lao O, Bobillo C, van Der Gaag K, et al. (2010). Inferring continental ancestry of argentineans from Autosomal, Y-chromosomal and mitochondrial DNA. Ann. Hum. Genet. 74: 65-76.

Hofmann MH, Blievernicht JK, Klein K, Saussele T, et al. (2008). Aberrant splicing caused by single nucleotide polymorphism c.516G>T [Q172H], a marker of CYP2B6*6, is responsible for decreased expression and activity of CYP2B6 in liver. J. Pharmacol. Exp. Ther. 325: 284-292.

Marzolini C, Telenti A, Decosterd LA, Greub G, et al. (2001). Efavirenz plasma levels can predict treatment failure and central nervous system side effects in HIV-1-infected patients. AIDS 15: 71-75.

Mastrogianni O, Gbandi E, Orphanidis A, Raikos N, et al. (2014). Association of the CYP2B6 c.516G>T polymorphism with high blood propofol concentrations in women from northern Greece. Drug Metab. Pharmacokinet. 29: 215-218.

Nyakutira C, Roshammar D, Chigutsa E, Chonzi P, et al. (2008). High prevalence of the CYP2B6 516G->T( $\left.{ }^{*} 6\right)$ variant and effect on the population pharmacokinetics of efavirenz in HIVIAIDS outpatients in Zimbabwe. Eur. J. Clin. Pharmacol. 64: $357-365$. 
Panel on Antiretroviral Guidelines for Adults and Adolescents 2012. (2013). Guidelines for the use of antiretroviral agents in HIV-1-infected adults and adolescents. Department of Health and Human Services, Washington, DC. http://aidsinfo.nih. gov/ContentFiles/AdultandAdolescentGL.pdf. Accessed December 21. 2013.

Raccor BS, Claessens AJ, Dinh JC, Park JR, et al. (2012). Potential contribution of cytochrome P450 2 B6 to hepatic 4-hydroxycyclophosphamide formation in vitro and in vivo. Drug Metab. Dispos. 40: 54-63.

Ramachandran G, Hemanth Kumar AK, Rajasekaran S, Kumar P, et al. (2009). CYP2B6 G516T polymorphism but not rifampin coadministration influences steady-state pharmacokinetics of efavirenz in human immunodeficiency virus-infected patients in South India. Antimicrob. Agents Chemother. 53: 863-868.

Wang $\mathrm{H}$ and Tompkins LM (2008). CYP2B6: new insights into a historically overlooked cytochrome P450 isozyme. Curr. Drug Metab. 9: 598-610.

1000 Genomes Project Consortium, Abecasis GR, Auton A, Brooks LD, et al. (2012). An integrated map of genetic variation from 1,092 human genomes. Nature 491: 56-65. 